\title{
OBIA System for Identifying Mesoscale Oceanic Structures in SeaWiFS and MODIS-Aqua Images
}

\author{
Eva Vidal-Fernández, Jose A. Piedra-Fernández, Jesús M. Almendros-Jiménez, and Manuel Cantón-Garbín
}

\begin{abstract}
The ocean covers over $70 \%$ of the surface of our planet and plays a key role in the global climate. Most ocean circulation is mesoscale (scales of 50-500 $\mathrm{km}$ and 10-100 days), and the energy in mesoscale circulation is at least one order of magnitude greater than general circulation; therefore, the study of mesoscale oceanic structures (MOS) is crucial to ocean dynamics, making it especially useful for analyzing global changes. The detection of MOS, such as upwellings or eddies, from satellites images is significant for marine environmental studies and coastal resource management. In this paper, we present an objectbased image analysis (OBIA) system which segments and classifies regions contained in sea-viewing field-of-view sensor (SeaWiFS) and Moderate Resolution Imaging Spectro-radiometer (MODIS)Aqua sensor satellite images into MOS. After color clustering and hierarchical data format (HDF) file processing, the OBIA system segments images and extracts image descriptors, producing primary regions. Then, it merges regions, recalculating image descriptors for MOS identification and definition. First, regions are labeled by a human-expert, who identifies MOS: upwellings, eddies, cool, and warm eddies. Labeled regions are then classified by learning algorithms (i.e., decision tree, Bayesian network, artificial neural network, genetic algorithm, and near neighbor algorithm) from selected features. Finally, the OBIA system enables images to be queried from the user interface and retrieved by means of fuzzy descriptors and oceanic structures. We tested our system with images from the Canary Islands and the North West African coast.
\end{abstract}

Index Terms-Automatic recognition, fuzzy logic, image retrieval, moderate resolution imaging spectro-radiometer (MODIS), object-based image analysis (OBIA), ocean satellite images, sea-viewing field-of-view sensor (SeaWiFS).

\section{INTRODUCTION}

$\mathbf{O}$ BJECT-BASED image analysis (OBIA) is a recently proposed technique [1] used to analyze satellite images. It focuses on semantic information, partitioning remote sensing imagery into meaningful image objects and setting their relationships using contextual and spectral information [2]. The purpose of OBIA systems is to automatically or semiautomatically emulate the interpretation of a human-expert. Nevertheless, the success of object-based classification strongly

Manuscript received June 16, 2014; revised January 25, 2015; accepted January 26, 2015. Date of publication March 02, 2015; date of current version March 27, 2015. This work was supported in part by the EU ERDF and the Spanish Ministry of Economy and Competitiveness (MINECO) under Projects TIN2013-41576-R and TIN2013-44742-C4-4-R, and CGL2013-48202$\mathrm{C} 2-2-\mathrm{R}$, in part by the Andalusian Regional Government (Spain) under Project P10-TIC-6114, and in part by the CEiA3 and CEIMAR consortiums.

The authors are with the Department of Informatics, University of Almería, 04120 Almeria, Spain (e-mail: jalmen@ual.es).

Color versions of one or more of the figures in this paper are available online at http://ieeexplore.ieee.org.

Digital Object Identifier 10.1109/JSTARS.2015.2400223 depends on the quality of segmentation stage results [3], as explained below.

The study of mesoscale oceanic structures (MOS) is crucial for ocean dynamics, making it especially useful for analyzing global change [4]-[7]. Mesoscale refers to the range of phenomena in a predominant spatial scale approximately one to ten times the baroclinic Rossby radius with a concomitant time scale of one to ten inertial periods. This encompasses approximate ranges of 10 to several hundred kilometers and time periods of 1 day to several weeks [8]. Color ocean satellite images provide a large-scale view of biological processes. Sea-viewing field-of-view sensor (SeaWiFS) of chlorophyll-a (OC) and Moderate Resolution Imaging Spectro-radiometer (MODIS)-Aqua of chlorophyll-a and sea surface temperatures (SST) are an important resource for the study of the ocean environment [9]-[11].

The OBIA systems [1] go further than classical techniques in which the classification is carried out after segmentation. The main goal is to find objects of interest from primary segmented objects, by employing the knowledge of a human-expert [12]. It also involves the use of specific segmentation techniques for each object class [13]. In our case, merging takes into account the particular characteristics of each MOS and the knowledge of human-experts on the subject.

The morphological and contextual variability of the MOS make the use of OBIA systems crucial. Some authors have already used OBIA systems for analyzing scenarios with high spatio-temporal variability [14]-[16]. We have a wide time range with images from 1997 to 2013 with clouds partly covering MOS.

The OBIA satellite imaging techniques [1] make use of a set of descriptors enabling objects of interest in images to be described and labeled. Low-level descriptors (area, centroid, invariant moments, etc.) give morphologic descriptions of an object as well as its location. Nevertheless, they do not give enough contextual information, and more importantly, they do not deal with spectral information (temperature and chlorophyll levels compared to the surroundings), which is crucial for MOS identification [17]. The expert knowledge of oceanographers is therefore required. Furthermore, because fuzzy logic is the best choice for overcoming this problem [18], we use fuzzy descriptors in our OBIA system to be able to include the knowledge of a human-expert's identification of MOS.

The use of ontologies for ocean satellite image classification is studied in [19] and their practical use for handling elements present in images and MOS is shown. Some other publications [20], [21] use ontologies to solve semantic mismatching 


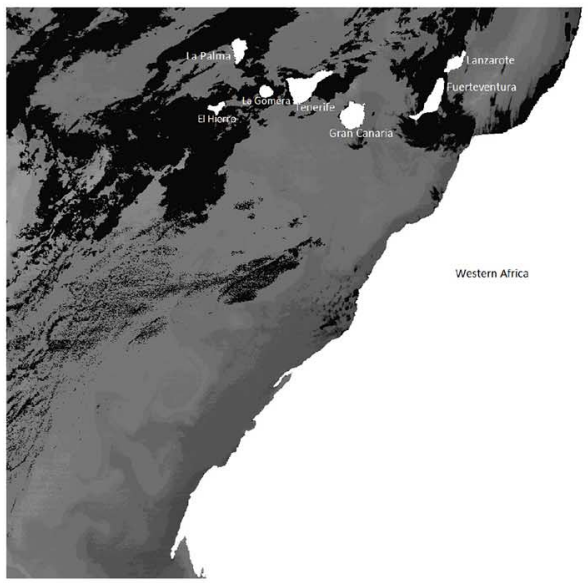

(a)

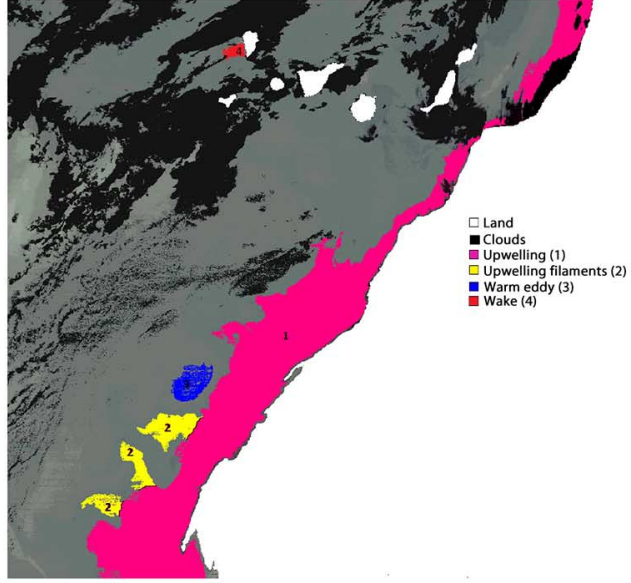

(b)

Fig. 1. MODIS-Aqua-SST scene (2003-03-24). (a) Original SST image in grayscale. (b) Ocean structure map where upwellings have been colored in pink for visualization (1), upwelling filaments (Cape Blanc) in yellow (2), warm eddies in blue (3), and wakes (west of La Palma) in red (4).

and heterogeneity in this field. We make use of ontologies for natural descriptions of MOS, and they are mainly used in the retrieval stage to provide the human-expert with a common language.

Many studies have reveal that SeaWiFS (OC) and MODISAqua-OC sensor chlorophyll images and MODIS-Aqua-SST temperature sensor images are a suitable choice for MOS [7], [9]-[11], primary production [5], and global change [4] any place in the world. In [6], the authors analyze data from chlorophyll images for a 10-year period, comparing them with data collected in situ. They also compare results with different sensors, finding highly correlated coefficients. In our work, we used images from three sensors (SeaWiFS, MODIS-Aqua-OC, and MODIS-Aqua-SST sensors) and analyzed the MOS identified, finding the advantages of some types of images over others for each type of structure.

This paper presents an OBIA system for segmenting and classifying regions in SeaWiFS and MODIS-Aqua sensor satellite images into MOS. The system is able to process thousands of images, identifying MOS in them. The system facilitates batch loading of images for this purpose, decompressing and extracting information from HDF files. An essential element of the OBIA system is the definition of an MOS ontology for two reasons. First, the ontology provides descriptors for MOS: upwelling, wakes, eddies, etc. Secondly, the ontology assigns (clusters of) colors to the MOS names. After a preprocessing stage (i.e., smoothing filter and histogram equalization), the OBIA system segments images (i.e., thresholding and edge detection), and extracts image descriptors, automatically forming primary regions. Region descriptors are spectral, morphological, and contextual features which we describe using a fuzzy ontology. The ontology includes fuzzy descriptors for vague concepts like big, small, cold, far, near, etc. Then it merges regions, recalculating image descriptors. The training process from merged regions and selected descriptors uses learning algorithms (C 4.5, Naive-Bayes, MLP, K-NN, and $N N g e$ ). Finally, the OBIA system enables images to be queried from the user interface and retrieved by means of fuzzy and MOS ontologies.

\section{A. Ocean Phenomena}

Our study area is in the Canary Islands and northwest coast of Africa (see Fig. 1). In this area, there are many MOS throughout the year. Many works have described the MOS in this area [8], [22]-[25].

A coastal upwelling is mainly due to wind that causes cold bottom water to reach the surface. They occur when dense cold water at the bottom of the ocean rises to the surface near the coast, transporting nutrients, and increasing the development and proliferation of phytoplankton. The water is colder on the bottom than surface water and rich in nutrients. Typically, the transition between the upwelling and other surface water is often sharp [8]. These upwellings occur regularly along the North West African coast (see Fig. 1) and others like the Peruvian, Californian, and SouthAfrican coasts, among others, where wind conditions are suitable. The analysis and prediction of upwellings, for which satellite images are also a powerful tool, are very important to commercial fishing.

There are some mesoscale cross-shore structures along the upwelling front called upwelling filaments which are tongueshaped cold upwellings. They are important coast to the open ocean nutrient carriers often found near capes [26] (e.g., off Cape Ghir or Cape Blanc) (see Fig. 1).

Eddies are highly morphologically and contextually variable structures. They may appear rounded near islands or in the open ocean. In cool eddies, cold nutrient-rich water rises to the surface [25], while warm eddies (see Fig. 1) drag water with organic material to the ocean floor and keep in the warm surface. Eddy water differs from the surrounding water in salinity and temperature and can also travel long distances for long periods of time without mixing with the surrounding water [23], [24]. The cool (cyclonic) eddies are created by calm wind conditions, while warm (anticyclonic) eddies are formed under stronger wind conditions.

Wakes are warm oceanic structures associated with islands [8], [23]. In our study area, these structures are generated by the obstacle formed by the Canary Islands and the predominant 


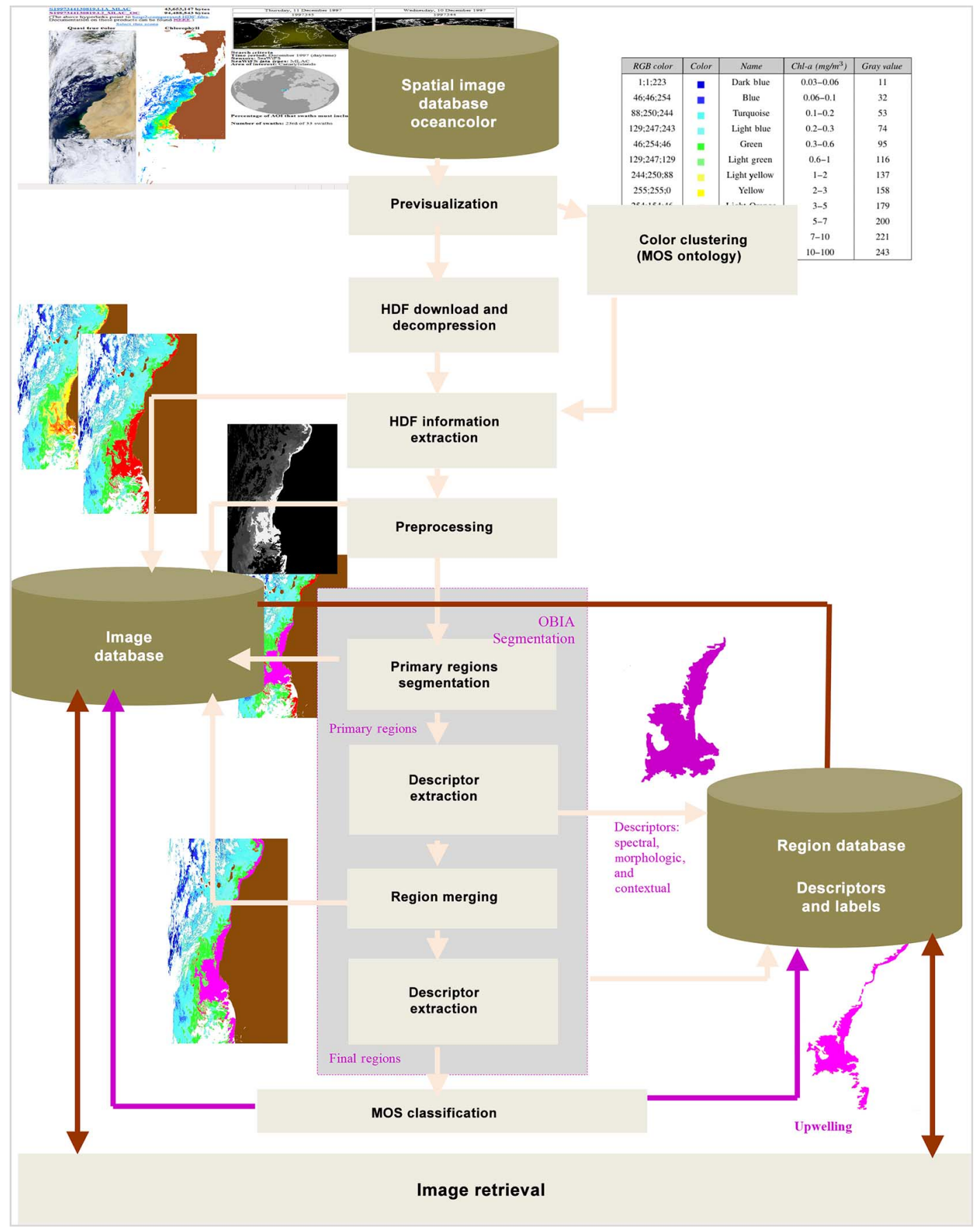

Fig. 2. OBIA system components.

NE winds. This interaction causes very thin tongues of hot, nutrient poor water (as compared to the surrounding water) to form close to the southwest islands (see Fig. 1). These structures are shown in Fig. 1(a) in an equalized MODIS-Aqua SST-scene and are classified in Fig. 1(b).

\section{B. Structure of the Paper}

Section II describes the OBIA system: image processing, descriptor extraction, merging, classification, and retrieval of regions and the main implementation elements. Section III analyzes the classification results, and in addition, a comparison of different sensors and classifiers is included. Finally, conclusion and suggestions for further improvement are presented in Section IV.

\section{OBIA SYSTEM}

In this paper, we present the OBIA system developed in our research group. The OBIA system input is satellite images from a public server. For our study area, we used the ocean color website [27]. The output is a set of labeled objects from the satellite images. The OBIA system structure is shown in Fig. 2. The general process is divided into several steps. 
1) Previsualization and $H D F$ processing: Analyzes $\mathrm{HDF}$ files and verifies the cloudiness in the study area. It includes download, decompression, and information extraction from HDF files.

2) Color clustering: Sets up the MOS ontology-MOS names and color clusters are mapped to each MOS name. The output of the color clustering is a color table.

3) Preprocessing: A set of image quality improvement techniques.

4) Segmentation: The process dividing the image into regions of interest. This includes region merging and descriptor extraction. A human-expert selects primary regions and the system merges and recalculates descriptors.

5) MOS classification: The human-expert labels the regions with one of the MOS ontology names. Learning algorithms are used for classification from selected descriptors.

6) Image retrieval: The processed images can be queried using the MOS and Fuzzy ontology concepts as vocabulary.

Fig. 2 also shows that our OBIA system handles the following databases.

1) Spatial image database: The HDF input data.

2) Region database: This includes region data-spectral, morphological and contextual features, the assigned labels, and information about the original and processed images. Some features are fuzzy descriptors which represent SST and OC level as well as size and distance to continent and islands.

3) Image database: Stores preprocessed, segmented and original images.

These steps are describe in more detail below.

\section{A. Color Clustering}

The human-expert defines the MOS ontology. Basically, starting from a table of color names in the image, he selects colors and assigns them to an MOS name. The MOS ontology consists of mapping color clusters to MOS names. MOS names are used in the image retrieval stage.

For instance, Table $\mathrm{I}$ is the original color table used in SST MODIS sensor images. The RGB color/color column is assigned to a temperature interval in the temperature column. Fig. 3 shows examples of color clustering. The choice of the best color cluster in this case depends on the ocean temperature, which is represented by the colors in the image. Fig. 3 shows an example of cold water. In this case, clustering from light lilac to lime green, which identifies colors in Table I from light lilac to lime green, i.e., image processing considers this whole range of color as dark blue [Fig. 3(c)] merges the upwelling with the surrounding water. Clustering from light lilac to dark purple, considers this whole range of color as dark purple [Fig. 3(d)] is a good option, but cannot detect the upwelling as a whole region. Pairwise clustering, which means that consecutive colors are identified two by two [Fig. 3(e)] is the best. The other MOS, which are wakes, are also detected.
TABLE I

ORIGINAL MODIS SENSOR SST IMAGE Color TABle

\begin{tabular}{|c|c|c|c|c|}
\hline RGB color & Color & Label & Temp. $\left({ }^{\circ} \mathrm{C}\right)$ & Gray value \\
\hline $208: 169: 245$ & $\square$ & Light lilac & $<11$ & 12 \\
$190 ; 129 ; 247$ & $\square$ & Lilac & $11-12$ & 12 \\
$154 ; 46 ; 254$ & $\square$ & Dark lilac & $12-13$ & 24 \\
$128 ; 0 ; 255$ & $\square$ & Blueing lilac & $13-14$ & 36 \\
$56 ; 11 ; 97$ & $\square$ & Dark purple & $14-15$ & 48 \\
$0 ; 0 ; 255$ & $\square$ & Dark blue & $15-16$ & 60 \\
$88 ; 88 ; 250$ & $\square$ & Blue & $16-17$ & 72 \\
$0 ; 128 ; 255$ & $\square$ & Light blue & $17-18$ & 84 \\
$0 ; 255 ; 255$ & $\square$ & Turquoise & $18-19$ & 96 \\
$0 ; 255 ; 128$ & $\square$ & Green (lime) & $19-20$ & 108 \\
$11 ; 97 ; 11$ & $\square$ & Dark green & $20-21$ & 120 \\
$4 ; 180 ; 4$ & $\square$ & Green (pigment) & $21-22$ & 132 \\
$0 ; 255 ; 0$ & $\square$ & Green & $22-23$ & 144 \\
$88 ; 250 ; 88$ & $\square$ & Light green & $23-24$ & 156 \\
$128 ; 255 ; 0$ & $\square$ & Green (lemon) & $24-25$ & 168 \\
$255 ; 255 ; 0$ & $\square$ & Yellow & $25-26$ & 180 \\
$244 ; 250 ; 88$ & & Yellow (egg) & $26-27$ & 192 \\
$250 ; 172 ; 88$ & $\square$ & Light orange & $27-28$ & 204 \\
$255 ; 128 ; 0$ & $\square$ & Orange & $28-29$ & 216 \\
$255 ; 0 ; 0$ & $\square$ & Red & $29-30$ & 228 \\
$138 ; 8 ; 8$ & $\square$ & Garnet & $>31$ & 228 \\
\hline
\end{tabular}

We have shown some clustering colors, but the system can create as many as the human-expert requires, and therefore, results are optimal for each case.

\section{B. Preprocessing}

This step improves image quality. For this purpose, noise (i.e., wrong pixels and small regions) is removed. The smoothing filter uses a double median filter applied to the grayscale image. The mean gray values for each range are shown in Table I. This reduces the computational cost. The output is a preprocessed BMP grayscale image stored in the image database.

\section{Segmentation}

1) Primary Region Segmentation: The image is segmented into primary regions using classical segmentation techniques [28] such as thresholding and edge detection. Some of these regions are labeled directly, but others, especially the upwellings, are merged in the region merging step (see Fig. 2). The input image is a gray scale image. The thresholding algorithm returns an image in which region pixels represent an interval. Then, a morphological opening algorithm is applied to smooth the contour, remove holes, and separate regions. After thresholding, edge detection is applied to recover the contour of the regions and their contents. The output is a segmented BMP image with primary regions, stored in the image database.

2) Descriptor Extraction: A number of numerical, spectral (OC and SST), morphological (size), and contextual (distance) descriptors are calculated for each primary region segmented. Some of these are fuzzy descriptors. The main morphological descriptors (also called low-level features) are shown in Table II. Some of the morphological descriptors (invariant moments, quotient of major and minor bounding ellipse axis, etc.) are invariants of affine transformations. As a result, the regions can be recognized despite rotations, translations, or 


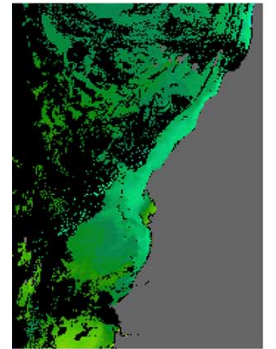

(a)

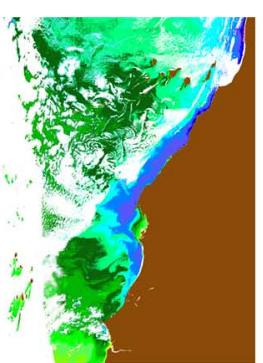

(b)

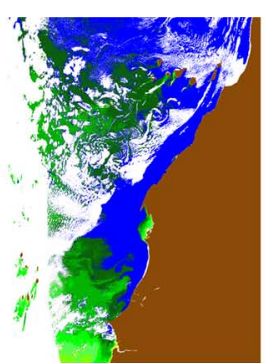

(c)

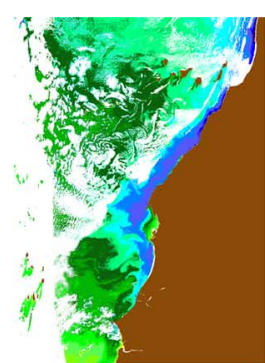

(d)

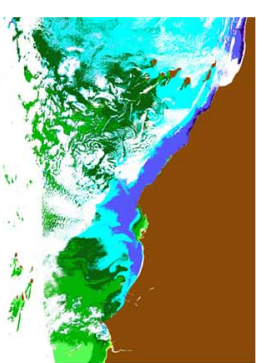

(e)

Fig. 3. Color clustering applied to MODIS-SST image (2009-05-18). (a) Ocean color website image. (b) Image colored using the original color table. (c) "From light lilac to lime green." (d) "From light lilac to dark purple." (e) Pairwise clustering.

TABLE II

LOW-LEVEL REGION DESCRIPTORS

\begin{tabular}{|c|c|c|}
\hline Category & Descriptor & Description \\
\hline Simple & $\begin{array}{l}\text { Area } \\
\text { Perimeter } \\
\text { Circularity }\end{array}$ & $\begin{array}{l}\text { Number of pixels } \\
\text { Number of contour } \\
\text { pixels } \\
\text { Degree of circular- } \\
\text { ity ( } 1 \text { is rounded) }\end{array}$ \\
\hline BoundingEllipse & $\begin{array}{l}\text { MajorAxis } \\
\text { MinorAxis } \\
\text { Orientation } \\
\text { Circumscribed } \\
\\
\text { Eccentricity }\end{array}$ & $\begin{array}{l}{[29]} \\
{[29]} \\
\text { Rotation angle } \\
\text { Value of zero means } \\
\text { ellipse is out } \\
\text { and one means el- } \\
\text { lipse is completely } \\
\text { on the region } \\
\text { Measure of how } \\
\text { much the conic } \\
\text { section deviates } \\
\text { from being circular }\end{array}$ \\
\hline BoundingBox & $\begin{array}{l}\text { BoundingBox_width } \\
\text { BoundingBox_height } \\
\text { BoundingBox_coord_x } \\
\text { BoundingBox_coord_y }\end{array}$ & $\begin{array}{l}\text { Width of the bound- } \\
\text { ing box } \\
\text { Height of the } \\
\text { bounding box } \\
\text { Coordinate } X \text { of the } \\
\text { first point } \\
\text { Coordinate } Y \text { of of } \\
\text { the first point }\end{array}$ \\
\hline Centroide & $\begin{array}{l}\text { Centroidex } \\
\text { Centroidey }\end{array}$ & $\begin{array}{l}\text { Coordinate } X \text { of the } \\
\text { centroid point } \\
\text { Coordinate } Y \text { of the } \\
\text { centroid point }\end{array}$ \\
\hline HuMoment & $\begin{array}{l}\text { Hu1, Hu2, Hu3, Hu4, Hu5, } \\
\text { Hu6, Нu7 }\end{array}$ & {$[30]$} \\
\hline Maitra moment & $\begin{array}{l}\text { MM1, MM2, MM3, MM4, } \\
\text { MM5, MM6 }\end{array}$ & {$[31]$} \\
\hline Tensorial moment & $\begin{array}{l}\text { MT00, MT01, MT10, MT11, } \\
\text { MT12, MT20, MT21, MT02, } \\
\text { MT30, MT03 }\end{array}$ & [32] \\
\hline Zernike moment & $\begin{array}{l}\text { MZ1, MZ2, MZ3, MZ4, MZ5, } \\
\text { MZ6, MP1, MP2 }\end{array}$ & [33] \\
\hline
\end{tabular}

scale changes over the image. These descriptors are stored in the region database along with the processed image data (name, date of capture, sensor, etc.).

There are a number of fuzzy descriptors, which are essential to the region merging phase and also serve as the query language for image retrieval. These fuzzy descriptors define a fuzzy ontology of vague concepts. The fuzzy descriptors are shown in Table III. Each fuzzy descriptor has a number of vagueness values. For instance, distance to island can be near, medium, or far. Each value has a degree of membership, i.e., a certain region is
TABLE III

FUZZY ONTOLOGY

\begin{tabular}{|l|l|}
\hline \multicolumn{1}{|c|}{ Feature } & \multicolumn{1}{c|}{ Vague concepts } \\
\hline Chl-a & Very low, low, medium, high, very high \\
SST & Very cold, cold, hot, warm, very hot \\
Distance to continent & Near, medium, far \\
Distance to island & Near, medium, far \\
Region size & Small, medium, large \\
\hline
\end{tabular}

near an island by a certain degree (i.e., a value of $[0,1]$ interval). Degree of membership is defined by a triangular distribution.

3) Region Merging: The human-expert selects the primary regions to be merged. He can use fuzzy descriptors to find them. A region bounding box is used to merged the regions. The largest region contained in the bounding box is called the base region, as shown in Fig. 4(a). This is followed by an iterative process which starts by merging the base region with another region and rebuilds the bounding box, forming a new one that includes both. The bounding box keeps growing until no more regions can be merged in it. The resulting region is the merged region. All descriptors are recalculated for this new region and stored in the region database, as well as the BMP image with the merged region, which is stored in the image database.

It should be mentioned that a set of valid merged regions are needed for identifying MOS. This requires optimal initial segmentation into primary regions, i.e., a small number of solid well-defined regions. It facilitates regions growing into significant merged regions and identification of MOS from the surrounding ocean.

Fig. 4 shows an example of region merging in a SeaWiFS image (2000-12-11). The upwellings here are very large structures, which are often partially covered by clouds that fragment the region. Whenever these primary regions are close to each other, they can be merged into one new region which will be labeled as an MOS. In this example, primary regions are colored yellow, while the merged region is colored by the system in pink for emphasis.

\section{MOS Classification}

We have selected several classifiers based on their low computational cost and good results reported in the literature. The selected classifiers are decision tree, Bayesian network, artificial neural network, K-nearest neighbor algorithm, and improved K-NN algorithm based on genetic algorithms. The C4.5 algorithm generates a decision tree developed by 


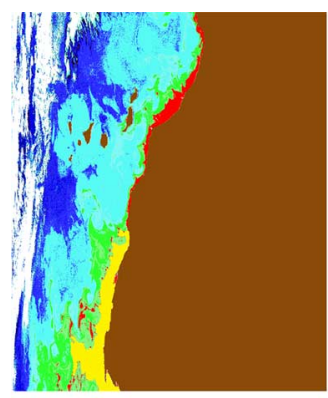

(a)

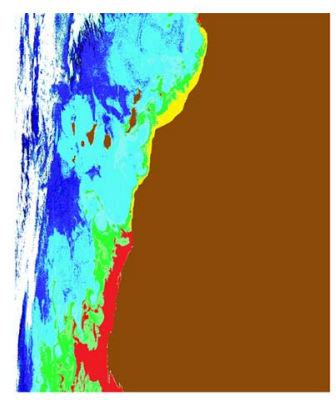

(b)

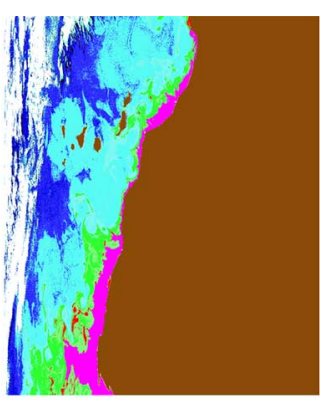

(c)

Fig. 4. Region merging. SeaWiFS image (2000-12-11). (a) Primary region 1 (yellow) part of upwelling close to the islands. (b) Primary region 2 (yellow) part of upwelling. (c) Merged region (pink) whole upwelling.

TABLE IV

LABELED MOS AND SENSORS

\begin{tabular}{|c|c|c|c|c|}
\hline MOS & SW & M-OC & M-SST & Total \\
\hline Upwelling & 122 & 79 & 83 & 284 \\
Upwelling filament & 23 & 14 & 7 & 44 \\
Cool eddy & 8 & 1 & 1 & 10 \\
Warm eddy & 2 & 1 & 1 & 4 \\
Wake & 3 & 1 & 19 & 23 \\
\hline
\end{tabular}

SW, SeaWiFS (OC); M-OC, MODIS-Aqua-OC; M-SST, MODIS-Aqua-SST.

Quinlan [34]. C4.5 builds decision trees from a set of training data, using entropy. The Naive Bayes classifier [35] is the simplest Bayesian network model. It assumes that given the class, all descriptors are independent of each other. Multilayer perceptron (MLP) [36] is an artificial multiple-layer neural network, which uses back-propagation to classify different regions. $K$-NN (K-nearest neighbors classifier) [37] assigns the region to its nearest neighbor's class. NNge [38] is a K-NN algorithm based on genetic algorithm non-nested generalized exemplars (which are hyper-rectangles used as if-then rules).

The numbers of labeled MOS for each sensor are summarized in Table IV. There are only a few cool eddies, warm eddies, and wakes because of the cloudiness in the study area and the size of the structure class.

Fig. 5 shows examples of segmented regions where the labeled regions are colored by the system in pink color for emphasis. Colors from yellow to red (considering this whole range of color as red) were clustered in the OC images (Fig. 5). These figures show how different MOS structures are. Upwellings in this area are large, elongated and close to the continent in both OC and SST images [Fig. 5(a)]. According to the color tables, upwellings in SST images are represented by blue-purple and dark green, while in OC images, upwellings are usually represented by red, except when they are emphasized in pink.

Upwelling filaments are elongated structures, starting from an upwelling and extending out to sea [Fig. 5(b)]. The color is usually the same as the color of the upwelling they come from, and although their size varies, they are usually medium-sized or small. Eddies are rounded and small. Cool eddies (cyclonics) have a higher chlorophyll concentration and are colder than the surrounding water [Fig. 5(c)]. Warm eddies (anticyclonic) have a lower chlorophyll concentration and are warmer than the surrounding water [Fig. 5(d)]. Wakes are similar to warm eddies but small, elongated and close to islands [Fig. 5(e)].

\section{E. Image Retrieval}

In this image retrieval step, the user can search for images in the image database by any of several different criteria, and the OBIA system retrieves them by means of the region database descriptors and information about images. Images can be retrieved by date, sensor, etc. More sophisticated queries use the MOS and fuzzy ontologies for the region and image databases. For instance, images containing a wake, an upwelling, etc., can be retrieved using the MOS ontology, or a query can ask for a wake in an OC image using the fuzzy ontology

$<<$ A Region with "Near an Island" membership greater than or equal to 0.5 and "Small" membership greater than or equal to $0.7>>$

$<<$ A Region with "Low chlorophyll concentration" membership greater or equal to 0.4 or "Very low chlorophyl concentration" membership greater than or equal to $0.1>>$

\section{F. OBIA Implementation}

The OBIA system was implemented using the Java, C, and SQL programming languages. In particular,

1) Java is used for implementing the user interface as well as for handling HDF. Java Swing and HDF libraries are used for this.

2) SQL is embedded in Java by means of the Java SQL library.

3) $\mathrm{C}$ is used to perform image processing by means of the OpenCV library.

The user interface (see Fig. 6) includes a menu that creates Java panels with different elements.

Once an image is selected, a query interface panel appears [see Fig. 6(a)]. The query interface makes it possible to retrieve regions by means of fuzzy descriptors via SQL. Merging and labeling are possible for selected regions [see Fig. 6(b)]. Finally, the system also enables the image database to be queried for comparing MOS from different sensors.

\section{ANALYSIS AND ReSUlts}

We tested our OBIA system with $\mathbf{2 1 2}$ satellite images of the Canary Islands and the Northwest African coast (the geographic 


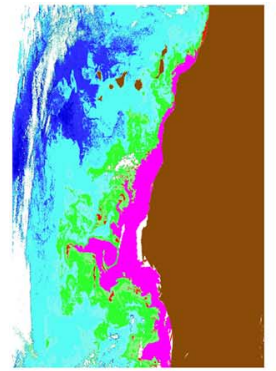

(a)

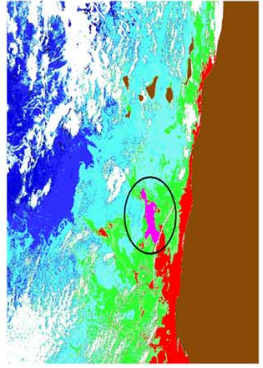

(b)

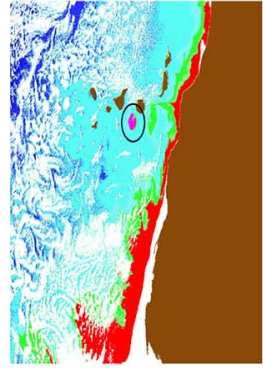

(c)

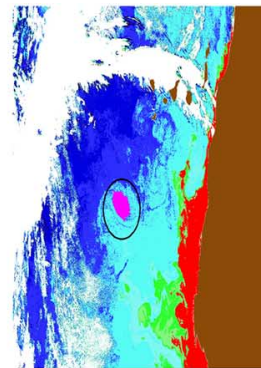

(d)

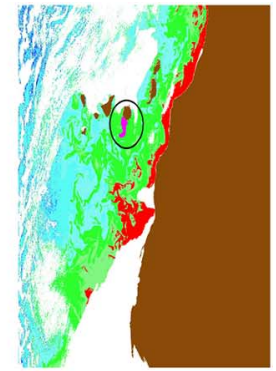

(e)

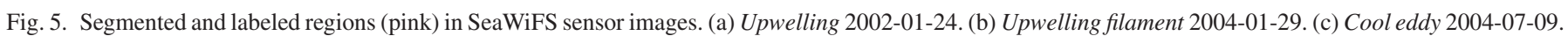
(d) Warm eddy 2004-11-22. (e) Gran Canaria island wake 2004-03-17.

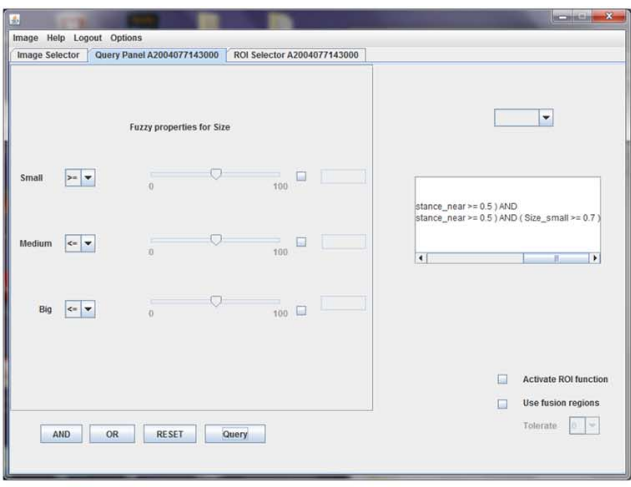

(a)

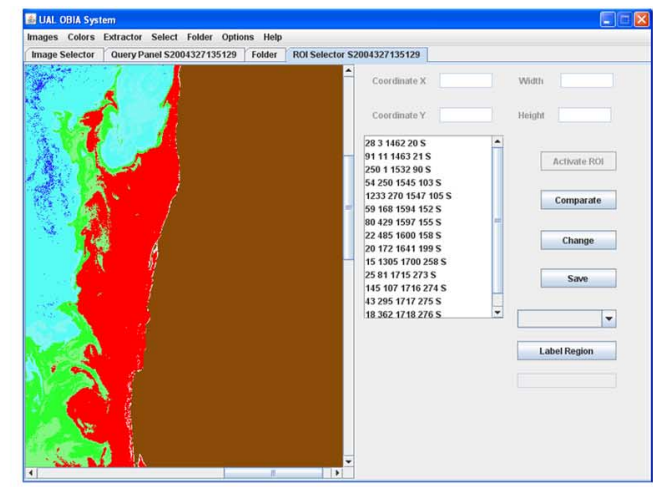

(b)

Fig. 6. OBIA system. (a) Query panel. (b) Merging and ableling apnel.

center of the Island is $28.2^{\circ} \mathrm{N}$ and $16.4^{\circ} \mathrm{W}$ ). Of them, 92 images are chlorophyll images [Chl-a quantity contained in the ocean $(O C)]$ adquired from the SeaWiFS sensor on board the Orbview-2 satellite (1.1 km resolution), for 1997-2004. These images are derived from merged local area coverage (MLAC) products [27]. The MLAC products consolidate all of the LAC from different receiving stations available for the same orbit, in geographic regions with multiple high resolution picture transmissions (HRPT) stations. Overlapping scenes are evaluated to adquire a single best image without duplication. The MLAC product was chosen because it generates high-quality L2 level images, including radiometric calibration and geometric correction. The other 120 images are from the MODIS-Aqua sensor on board the Aqua satellite (1.1 km resolution), for 2003-2013 and divided into 61 chlorophyll and 59 sea surface temperature (SST) images. These images are derived from L2 level local area coverage (LAC) products [27].

A total of $\mathbf{3 6 5}$ MOS were detected of which 284 MOS correspond to upwellings or parts of upwellings (when clouds keep parts of upwellings from being merged), 44 upwelling filaments, 10 cool eddies, 4 warm eddies, and 23 wakes.

We analyzed a batch of images taken by different sensors with our OBIA system, for each sensor, separately, and also by grouping regions into three categories: OC sensors (SeaWiFS and MODIS-OC), MODIS sensors (MODIS-OC and SST), and all together (SeaWiFS, MODIS-OC, and MODIS-SST). Table V shows the accuracy rates achieved by the different classification algorithms and Table VI summarizes the descriptors selected for each test. Tenfold cross-validation was used
TABLE V

MOS ClASSIFICATION (\%)

\begin{tabular}{|c|c|c|c|c|c|c|}
\hline Algorithm & SW & M-OC & M-SST & SWOC & MOD & ALL \\
\hline C 4.5 & 96.20 & 95.83 & 94.59 & 96.46 & 93.72 & 93.42 \\
NaiveBayes & 88.61 & 94.79 & 95.50 & 90.16 & 95.17 & 78.36 \\
MLP & 93.04 & 96.88 & 95.50 & 95.28 & 97.10 & 95.07 \\
K-NN (2) & 93.67 & 96.88 & 96.40 & 95.28 & 95.17 & 94.79 \\
NNge & 96.84 & 96.88 & 93.69 & 96.06 & 95.65 & 96.99 \\
\hline
\end{tabular}

SW, SeaWiFS (OC); M-OC, MODIS-Aqua-OC; M-SST, MODIS-AquaSST; SWOC, SeaWiFS (OC)+MODIS-Aqua-OC; MOD, MODIS-AquaOC+MODIS-Aqua-SST; ALL, OC+SST.

for all tests. A correlation-based feature selection algorithm (CFS) [39] was used for descriptor selection in classification. The CFS algorithm returns a descriptor ranking with a percentage of relevance. This percentage indicates the degree of relevance (around 100\%) or irrelevance (around 0\%) of the descriptor to classification. The selection criterion is to choose all the descriptors with over $60 \%$ relevance, in each case. It provides a good accuracy rate when there are a small number of features. The results of each test are given in Fig. 7-12. In each figure, we show the accuracy rate for each classifier and MOS. In our experiments, the use of the same descriptors reduces the accuracy rate in the classification process.

The SeaWiFS sensor test cases included 122 upwellings, 23 upwelling filaments, 8 cool eddies, 2 warm eddies, and 3 wakes. Fig. 7 shows that the NNge algorithm had the best accuracy rate of over $30 \%$, in all cases, including warm eddies and wakes (even though there were only a few wakes). The accuracy rate with this algorithm was $96.84 \%$. As expected, accuracy rates 
TABLE VI

SELECTED DESCRIPTORS

\begin{tabular}{|c|c|c|c|c|c|c|}
\hline Descriptor & SW & $\mathrm{M}-\mathrm{OC}$ & M-SST & SWOC & MOD & ALL \\
\hline $\begin{array}{c}\text { Perimeter } \\
\text { MajorAxis } \\
\text { MinorAxis } \\
\text { Orientation } \\
\text { Cirscumscribed }\end{array}$ & & $\begin{array}{l}\checkmark \\
\checkmark\end{array}$ & $\begin{array}{l}\checkmark \\
\checkmark\end{array}$ & $\checkmark$ & $\checkmark$ & $\checkmark$ \\
\hline $\begin{array}{l}\text { BBox_height } \\
\text { BBox_width }\end{array}$ & $\checkmark$ & $\begin{array}{l}\checkmark \\
\checkmark\end{array}$ & $\checkmark$ & $\checkmark$ & $\checkmark$ & $\checkmark$ \\
\hline $\begin{array}{l}\mathrm{Hu} 1 \\
\mathrm{Hu} 2 \\
\mathrm{Hu} 4\end{array}$ & $\checkmark$ & & & $\begin{array}{l}\checkmark \\
\checkmark\end{array}$ & $\checkmark$ & $\checkmark$ \\
\hline MM6 & & & & & & $\checkmark$ \\
\hline $\begin{array}{l}\text { MT11 } \\
\text { MT12 }\end{array}$ & & & $\checkmark$ & & $\begin{array}{l}\checkmark \\
\checkmark\end{array}$ & \\
\hline $\begin{array}{l}\text { MZ3 } \\
\text { MZ5 }\end{array}$ & & $\checkmark$ & & & & $\checkmark$ \\
\hline $\begin{array}{c}\text { Chl-a_very_low } \\
\text { Chl-a_low } \\
\text { Chl-a_medium } \\
\text { Chl-a_high }\end{array}$ & $\begin{array}{l}\checkmark \\
\checkmark\end{array}$ & $\checkmark$ & & $\begin{array}{l}\checkmark \\
\checkmark\end{array}$ & $\begin{array}{l}\checkmark \\
\checkmark\end{array}$ & $\begin{array}{l}\checkmark \\
\checkmark \\
\checkmark\end{array}$ \\
\hline $\begin{array}{c}\text { Temp_very_cold } \\
\text { Temp_cold } \\
\text { Temp_warm } \\
\text { Temp_hot }\end{array}$ & & & $\checkmark$ & & $\begin{array}{l}\checkmark \\
\checkmark\end{array}$ & $\begin{array}{l}\checkmark \\
\checkmark \\
\checkmark\end{array}$ \\
\hline $\begin{array}{l}\text { Cont_dis_near } \\
\text { Cont_dis_medium } \\
\text { Cont_dis_far }\end{array}$ & $\checkmark$ & $\begin{array}{l}\checkmark \\
\checkmark \\
\checkmark\end{array}$ & $\begin{array}{l}\checkmark \\
\checkmark\end{array}$ & $\begin{array}{l}\checkmark \\
\checkmark\end{array}$ & $\begin{array}{l}\checkmark \\
\checkmark\end{array}$ & $\checkmark$ \\
\hline $\begin{array}{c}\text { Isd_dis_near } \\
\text { Isd_dis_medium }\end{array}$ & $\checkmark$ & & $\checkmark$ & & $\checkmark$ & $\checkmark$ \\
\hline
\end{tabular}

SW, SeaWiFS (OC); M-OC, MODIS-Aqua-OC; M-SST, MODIS-AquaSST; SWOC, SeaWiFS (OC)+MODIS-Aqua-OC; MOD, MODIS-AquaOC+MODIS-Aqua-SST; ALL, OC+SST.

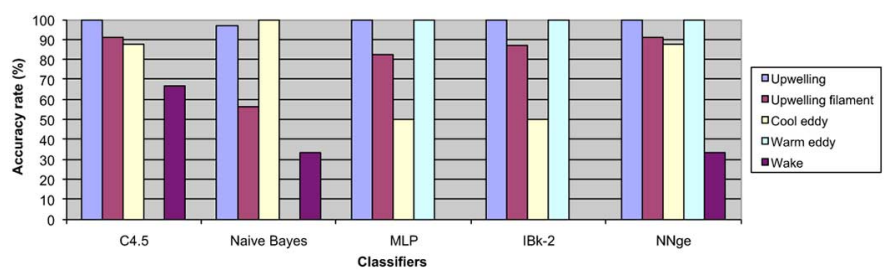

Fig. 7. Accuracy rates for SeaWiFS sensor (OC).

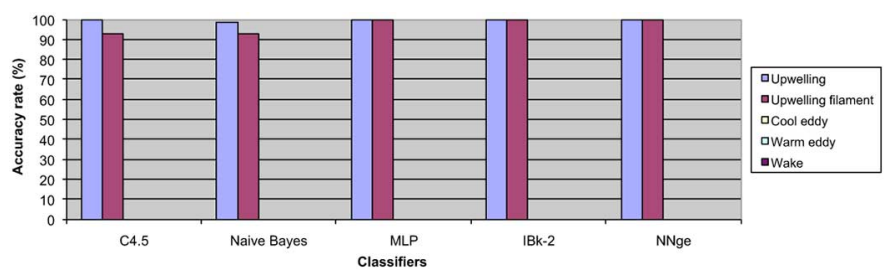

Fig. 8. Accuracy rates for MODIS-Aqua-OC sensor.

were best for upwellings (100\% in most cases). This is because upwellings are well differentiated from other structures, and because of the large number of test cases. Upwelling filaments also had a high accuracy rate (over $80 \%$ in most cases) for the same reasons as upwellings.

The MODIS-OC sensor test cases included 79 upwellings, 14 upwelling filaments, 1 cool eddy, 1 warm eddy, and 1 wake. In Fig. 8, the best accuracy rates were found with the MLP, K$\mathrm{NN}$, and NNge algorithms. In this case, the accuracy rate for cool eddies, warm eddies and wakes was $0 \%$ due to the number of test cases. The accuracy rate was $96.88 \%$. Again, upwellings

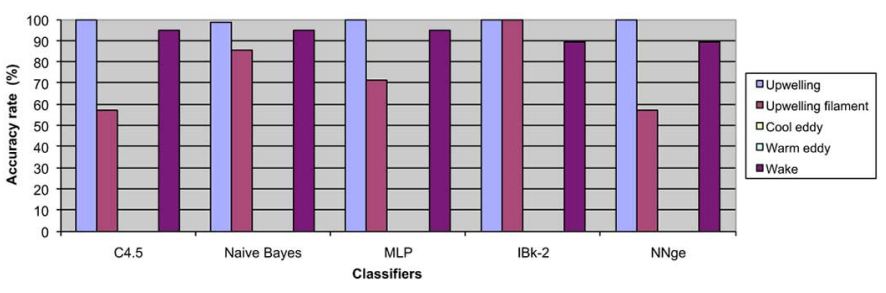

Fig. 9. Accuracy rates for MODIS-Aqua-SST sensor.

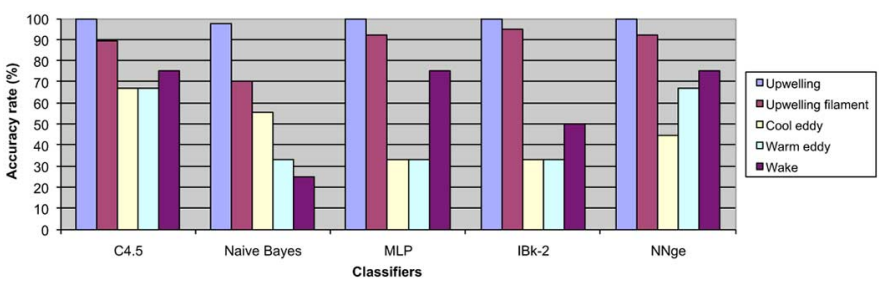

Fig. 10. Accuracy rates for SeaWiFS (OC) and MODIS-Aqua-OC sensors.

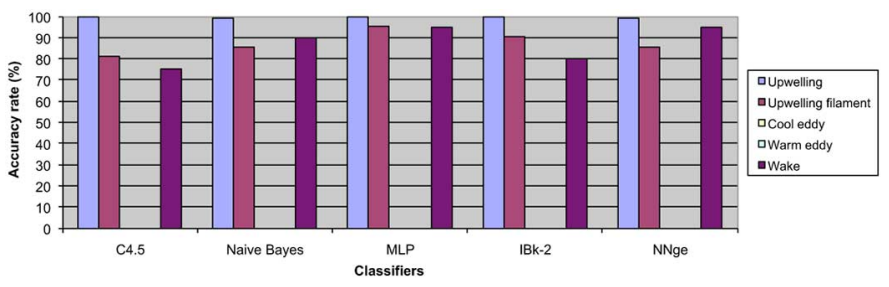

Fig. 11. Accuracy rates for MODIS-Aqua-OC and SST sensors.

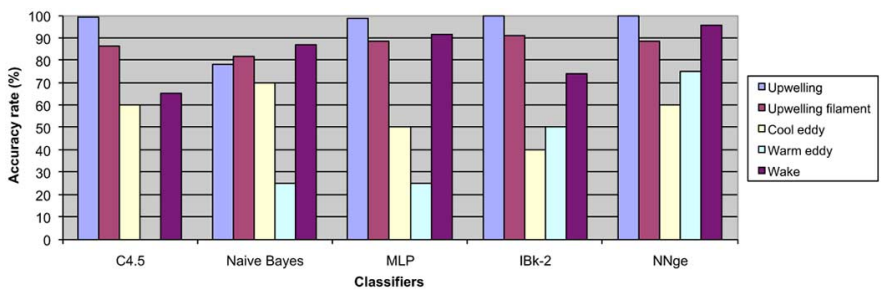

Fig. 12. Accuracy rates for SeaWiFS (OC), MODIS-Aqua-OC, and SST sensors.

had the best accuracy rate (100\% in most cases), followed by upwelling filaments (90\% in most cases).

The MODIS-SST sensor test cases included 83 upwellings, 7 upwelling filaments, 1 cool eddy, 1 warm eddy, and 19 wakes. In Fig. 9, the best accuracy rate was with the K-NN algorithm. Again, due to the number of eddies in the test cases, results were worse. The accuracy rate is $96.4 \%$. Upwelling accuracy rates are again the best. Here, wake rates outdid upwelling filaments (90\% in most cases).

The OC sensor (SeaWiFS and MODIS-OC) test cases included 201 upwellings, 37 upwelling filaments, 9 cool eddies, 3 warm eddies, and 4 wakes. In Fig. 10, the best accuracy rates were with the $\mathrm{C} 4.5$ algorithm, with over $65 \%$ in all cases, including warm eddies and wakes, even though there were only a few test cases. The accuracy rate was $96.46 \%$. This set of test cases is larger, making higher accuracy rates possible. Upwellings, upwelling filaments, and wakes have the top rates.

The MODIS OC/SST sensor test cases included 162 upwellings, 21 upwelling filaments, 2 cool eddies, 2 warm eddies, and 20 wakes. In Fig. 11, the best accuracy rate was with 
the MLP algorithm. For the same reasons as for the MODIS-OC and MODIS-SST tests, accuracy was the worst for eddies $(0 \%)$. The accuracy rate was $97.1 \%$ and over $90 \%$ for upwellings, upwelling filaments, and wakes in most cases.

The SeaWiFS and MODIS OC/SST sensor test cases included 284 upwellings, 44 upwelling filaments, 10 cool eddies, 4 warm eddies, and 23 wakes. In Fig. 12, the best accuracy rate of over $60 \%$ was with the NNge algorithm in all cases, including warm eddies (with a small number of test cases). The accuracy rate was $96.99 \%$. Since we used the full set of test cases here, the accuracy rates are over $60 \%$ in most cases, except for warm eddies, the smallest set of test cases, which had only $70 \%$ accuracy with the NNge algorithm.

From Table $\mathrm{V}$, the following conclusions may be arrived at. The accuracy rate is over $95 \%$ in most cases, and up to $97 \%$ in some cases. The best algorithm on the average is NNge (96.01\%). These test results reinforce our idea that improvement in segmentation by color clustering and region merging makes better automatic classification possible. Analyzing the descriptors we have selected (Table II), two of them are present in all the tests: BoundingBox_height and Continent_distance_near. Both are essential to MOS classification. Size and closeness to continent are crucial in distinguishing upwellings from other structures.

The results show that our system is able to process satellite images for regions labeling with a high accuracy rate. The MOS ontology not only provides an MOS vocabulary but also a set of color names to be clustered and mapped into one of the MOS.

In our study area, the upwelling is defined as a large elongated region, near a continent with a lower sea surface temperature and higher chlorophyll concentration than the surrounding sea. In most cases, upwelling candidates are fragmented by clouds, but segmentation based on region merging is able to rebuild the whole structure. In other cases, such as small regions (wakes or eddies), primary segmentation is enough.

Our system uses a relational database to store information about images (i.e., descriptors) and labeled objects in images. The system is equipped with a query interface that makes it possible to retrieve processed images by means of the MOS and fuzzy ontologies. Queries can be described in terms of the MOS ontology, or using vague fuzzy ontology concepts, enabling a degree of membership to be assigned to vague concepts. Images can be compared showing image features to be analyzed.

\section{CONCLUSion AND Future Work}

This paper presented an OBIA system that segments and classifies regions of interest contained in satellite SeaWiFS and MODIS-Aqua sensor images. An automatic process extracts segmented regions from images which are semi-automatically merged and labeled by a human-expert. Classification is achieved by learning algorithms. Ontologies are used for retrieval.

We have shown that there is a correlation between SST and OC images in MOS detection. The tests reveal that SST images are better for detecting wakes. For identifying upwellings, SST and OC both offer good results. For other MOS structures, the set of test cases should be larger for reliable conclusions.
Our aim was to improve image segmentation and, using humanexpert knowledge, the OBIA system is able to detect MOS structures regardless of whether SST or OC is used. In OC images, we are still unable to identify all the MOS, but those identified were reliable.

The system proposed has several weaknesses. The first one is that the number of images that has to be analyzed to find candidates for MOS identification may be quite large. For example, from an initial batch of 1000 images, only 400 may be valid. Our system includes a filter that discards images either outside of the area of study or off-center, and images with too much noise (e.g., clouds). The second is the effort required to obtain good segmentation from the color clustering selected. It forces segmentation to be iterated, clustering by several different colors. Successful segmentation should provide a small number of solid, well-defined primary regions in which regions are distinguished from the surrounding ocean.

For further research, we plan to include another large volume of data from other sensors to identify possible trends or changes in chlorophyll concentration or sea surface temperature by analyzing the morphology, movement, and evolution of MOS. In addition, we would like to predict MOS movement by analyzing their displacement, routing, and the time it takes for them to disappear. We would also like to use the system to analyze North Atlantic and North Pacific images that have the same ocean current and wind conditions, as well as MOS. Finally, we plan to fully automatize the system when a suitable training set is available.

\section{REFERENCES}

[1] T. Blaschke, "Object based image analysis for remote sensing," ISPRS J. Photogramm. Remote Sens., vol. 65, no. 1, pp. 2-16, 2010.

[2] B. N. Jyothi, G. Babu, and M. Krishna IV, "Object Oriented and multiscale image analysis: Strengths, weaknesses, opportunities and threatsA review," J. Comput. Sci., vol. 4, no. 9, pp. 706-712, 2008.

[3] I. A. Rizvi and B. K. Mohan, "Object-based image analysis of highresolution satellite images using modified cloud basis function neural network and probabilistic relaxation labeling process," IEEE Trans. Geosci. Remote Sens., vol. 49, no. 12, pp. 4815-4820, Dec. 2011.

[4] S. Sathyendranath et al., "Ocean colour climate change initiativeapproach and initial results," in Proc. IEEE Int. Geosci. Remote Sens. Symp. (IGARSS), 2012, pp. 2024-2027.

[5] B. Xiao, S. Hu, and X. Qiang, "Research on the ocean primary production pattern based remote sensing," in Proc. Int. Conf. Audio Lang. Image Process. (ICALIP), 2010, pp. 1543-1546.

[6] B. Saulquin, F. Gohin, and R. Garrello, "Regional objective analysis for merging high-resolution MERIS, MODIS/Aqua, and SeaWiFS chlorophyll-a data from 1998 to 2008 on the European Atlantic shelf," IEEE Trans. Geosci. Remote Sens., vol. 49, no. 1, pp. 143-154, Jan. 2011.

[7] Z. Liu and Y. Hou, "Kuroshio Front in the East China sea from satellite SST and remote sensing data," IEEE Geosci. Remote Sens. Lett., vol. 9, no. 3, pp. 517-520, Mar. 2012.

[8] E. D., Barton et al., "The transition zone of the Canary Current upwelling region," Prog. Oceanogr., vol. 41, no. 4, pp. 455-504, 1998.

[9] S. Patel, R. Balasubramanian, and A. Gangopadhyay, "Automatic detection of oceanic eddies in SeaWiFS-derived color images using neural networks and shape analysis," in Proc. IEEE Int. Geosci. Remote Sens. Symp. (IGARSS), 2008, vol. 2, pp. II-835-II-838.

[10] M. Mityagina and O. Lavrova, "Dynamic phenomena in the coastal waters of the north-eastern black sea retrieved from satellite data," in Proc. IEEE Int. Geosci. Remote Sens. Symp. (IGARSS'08), 2008, vol. 2, pp. II-347-II-350.

[11] J. Marcello, F. Marques, and F. Eugenio, "Automatic tool for the precise detection of upwelling and filaments in remote sensing imagery," IEEE Trans. Geosci. Remote Sens., vol. 43, no. 7, pp. 1605-1616, Jun. 2005. 
[12] M. Baatz, C. Hoffmann, and G. Willhauck, "Progressing from objectbased to object-oriented image analysis," in Object-Based Image Analysis. New York, NY, USA: Springer, 2008, pp. 29-42.

[13] M. Musci, R. Q. Feitosa, and G. A. Costa, "An object-based image analysis approach based on independent segmentations," in Proc. Joint Urban Remote Sens. Event (JURSE), 2013, pp. 275-278.

[14] P. Rastner, T. Bolch, C. Notarnicola, and F. Paul, "A comparison of pixeland object-based glacier classification with optical satellite images," IEEE J. Sel. Topics Appl. Earth Observ. Remote Sens., vol. 7, no. 3, pp. 853-862, Mar. 2014.

[15] L. Drăguț and T. Blaschke, "Automated classification of landform elements using object-based image analysis," Geomorphology, vol. 81, no. 3, pp. 330-344, 2006

[16] D. Jovanovic, M. Govedarica, I. Dordevic, and V. Pajic, "Object based image analysis in forestry change detection," in Proc. 8th Int. Symp. Intell. Syst. Informat. (SISY), 2010, pp. 231-236.

[17] J. A. Piedra-Fernandez, M. Cantón-Garbín, and J. Z. Wang, "Feature selection in AVHRR ocean satellite images by means of filter methods," IEEE Trans. Geosci. Remote Sens., vol. 48, no. 12, pp. 4193-4203, Nov. 2010.

[18] J. A. Piedra-Fernández, G. Ortega, J. Z. Wang, and M. Cantón-Garbín, "Fuzzy content-based image retrieval for oceanic remote sensing," IEEE Trans. Geosci. Remote Sens., vol. 52, no. 9, pp. 5422-5431, Mar. 2014.

[19] J. M. Almendros-Jiménez, L. Domene, and J. A. Piedra-Fernández, "A framework for ocean satellite image classification based on ontologies," IEEE J. Sel. Topics Appl. Earth Observ. Remote Sens., vol. 6, no. 2, pp. 1048-1063, May 2013

[20] S. R. Abidi, S. S. Abidi, M. Kwan, and A. Daniyal, "An ontology framework for modeling ocean data and E-science semantic web services," Int. J. Adv. Comput. Sci., vol. 2, no. 8, pp. 280-286, 2012.

[21] S. S. Durbha, R. L. King, and N. H. Younan, "An information semantics approach for knowledge management and interoperability for the global earth observation system of systems," IEEE Syst. J., vol. 2, no. 3, pp. 358365, Sep. 2008.

[22] L. García-Weil, M. Pacheco, G. Rodríguez, and T. McClimans, "Topographic effects on the mesoscale ocean circulation near Canarian Archipelago examined by means of satellite images and laboratory simulations," in Proc. IEEE Geosci. Remote Sens. Symp. (IGARSS), 2000, vol. 5, pp. 1830-1832.

[23] J. Arístegui et al., "Island-induced eddies in the Canary islands," Deep Sea Res. I: Oceanogr. Res. Papers, vol. 41, no. 10, pp. 1509-1525, 1994.

[24] P. Sangra et al., "Life history of an anticyclonic eddy," J. Geophys. Res., vol. 110 , no. C3, p. C03021, 2005 .

[25] A. Tejera, L. García-Weil, K. Heywood, and M. Cantón-Garbín, "Observations of oceanic mesoscale features and variability in the Canary Islands area from ERS-1 altimeter data, satellite infrared imagery and hydrographic measurements," Int. J. Remote Sens., vol. 23, no. 22, pp. 4897-4916, 2002.

[26] T. Meunier, E. D. Barton, B. Barreiro, and R. Torres, "Upwelling filaments off Cap Blanc: Interaction of the NW African upwelling current and the Cape Verde frontal zone eddy field," J. Geophys. Res.: Oceans, vol. 117 , no. C8, pp. 1-18, 2012.

[27] NASA. (2014). Ocean Color Web [Online]. Available: http://oceancolor. gsfc.nasa.gov

[28] G. Meinel and M. Neubert, "A comparison of segmentation programs for high resolution remote sensing data," Int. Arch. Photogramm. Remote Sens., vol. 35, no. Part B, pp. 1097-1105, 2004.

[29] J. Zunic and J. Slado, "Eficiency of characterizing ellipses and ellipsoids by discrete moments," IEEE Trans. Pattern Anal. Mach. Intell., vol. 22, no. 4, pp. 407-414, Apr. 2000.

[30] M.-K. Hu, "Visual pattern recognition by moment invariants," IRE Trans. Inf. Theory, pp. 179-187, Feb. 1962.

[31] S. Maitra, "Moment invariants," Proc. IEEE, vol. 67, no. 4, pp. 697-699, Apr. 1979.

[32] J. M. Galvez and M. Cantón, "Normalization and shape recognition of three-dimensional objects by $3 \mathrm{~d}$ moments," Pattern Recognit., vol. 26, no. 5 , pp. 667-681, 1993.

[33] M. R. Teague, "Image analysis via the general theory of moments," $J$. Opt. Soc. Amer., vol. 70, pp. 920-930, Aug. 1980.

[34] J. R. Quinlan, C4.5: Programs for Machine Learning. San Mateo, CA, USA: Morgan Kaufmann, 1993, vol. 1.

[35] G. H. John and P. Langley, "Estimating continuous distributions in Bayesian classifiers," in Proc. 11th Conf. Uncertainty Artif. Intell., 1995, pp. 338-345.
[36] M. L. Minsky and S. A. Papert, Perceptrons-Expanded Edition: An Introduction to Computational Geometry. Cambridge, MA, USA: MIT Press, 1987.

[37] D. W. Aha, D. Kibler, and M. K. Albert, "Instance-based learning algorithms," Mach. Learn., vol. 6, no. 1, pp. 37-66, 1991.

[38] B. Martin, "Instance-based learning: Nearest neighbour with generalisation," Ph.D. dissertation, Dept. Comput. Sci., Univ. Waikato, Hamilton, New Zealand, 1995

[39] M. A. Hall, "Correlation-based feature selection for machine learning," Ph.D. dissertation, Dept. Comput. Sci., Univ. Waikato, Hamilton, New Zealand, 1999.

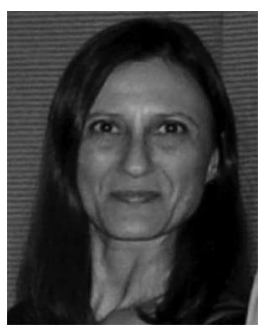

Eva Vidal-Fernández was born in Oviedo, in 1972. He received the Bachelor's degree in telecommunications from the University of Malaga, Malaga, Spain, and the M.S. degree in information security from Autonomous University of Madrid, Madrid, Spain, in 1997 and 2011, respectively. She is currently pursuing the Ph.D. degree at the University of Almería, Almera, Spain.

Her research interests include image retrieval, image processing, and soft computing.

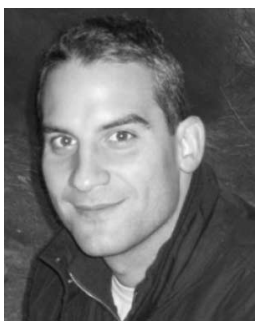

Jose A. Piedra-Fernández was born in Spain, in 1978. He received the Bachelor's, the M.S., and the $\mathrm{Ph} . \mathrm{D}$. degrees in computer Science all from Almería University, Almeria, Spain, in 2001, 2003, and 2005, respectively.

$\mathrm{He}$ is an Assistant Professor with Almería University. From 2008 to 2009, he was visiting the College of Information Sciences and Technology, Pennsylvania State University, State College, PA, USA. His research interests include image processing, pattern recognition, and image retrieval.

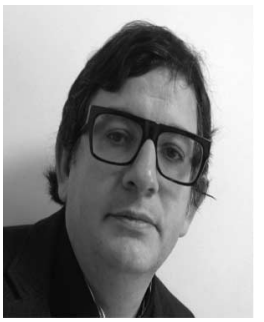

Jesús M. Almendros-Jiménez was born in Granada, in 1968. He received the Ph.D. degree in computer science from the Universidad Complutense de Madrid, Madrid, Spain, in 1999.

$\mathrm{He}$ is an Associate Professor with the Universidad de Almería. He is also the Leader of the Information Systems Group with the same university, and has been the Research-in-Chief of several research projects funded by the Spanish government. He is engaged in a project aiming to integrate Web technologies. His research interests include declarative languages, web databases, image retrieval, and model-driven development in software engineering.

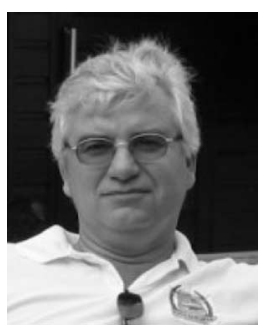

Manuel Cantón-Garbín was born in Adra (Abdera in phenician times) Almería, in 1955. He received the Bachelor's degree in physics (electronics) from Granada University, Spain, in 1979, and the M.S. degree in electronics and Ph.D. degree in pattern recognition from La Laguna University, Canary Islands, Spain, in 1980 and 1982, respectively.

In 1984, he moved to Las Palmas de Gran Canaria University (ULPGC), Canary Islands, Spain, and in 1986, he led the first group in Spain working on Satellite Oceanography. In 1986, he became a Lecturer and in 1991, a Full Professor in Applied Physics with ULPGC. In 1994, he moved to Almería University, where he is a Full Professor in Computing and Artificial Intelligence. His research interests include automatic ocean satellite images interpretation and analysis, and computer vision. 